LYCEN 9222

May 1992

\title{
Symmetry adaptation techniques in $n$-photon absorption spectroscopy
}

\author{
M. Daoud and $\underline{\text { M. Kibler }}$ \\ Institut de Physique Nucléaire, IN2P3-CNRS et Université Lyon-1, 69622 Villeurbanne \\ Cedex, France
}

\begin{abstract}
Communication to the International Conference Rare Earths' 92 in Kyoto, Kyoto, Japan, 1-5 June 1992 (contribution to "The Caro and Judd Symposium - Spectroscopy of Rare Earths").
\end{abstract}

Paper published in Journal of Alloys and Compounds 193 (1993) 219 (Proceedings of the International Conference Rare Earths' 92 in Kyoto). All correspondence concerning this paper should be addressed to : M. Kibler, Institut de Physique Nucléaire, Université Lyon-1, 43 Bd du 11 Novembre 1918, 69622 Villeurbanne Cedex, France. Telephone : (33) 724482 35. Fax : (33) 724480 04. (Electronic mail : kibler@frcpn11) 


\title{
Symmetry adaptation techniques in $n$-photon absorption spectroscopy
}

\author{
M. Daoud and M. Kibler
}

Institut de Physique Nucléaire, IN2P3-CNRS et Université Lyon-1, 69622 Villeurbanne Cedex, France

\section{Summary}

We present some recent progress achieved in the application of symmetry adaptation techniques to $n$-photon absorption spectroscopy of rare earth ions in finite symmetry. More specifically, this work is concerned with the determination of the intensity of $n$ photon transitions between Stark levels (rather than $J$ levels) of an ion in an environment with a given symmetry. The role of symmetry is taken into account through the use of initial and final state vectors characterized by irreducible representations of the (double) group for the ion site symmetry. Two distinct situations are considered, viz., the case of parity-allowed $n$-photon transitions (as e.g. intra-configurational transitions between Stark levels of the $4 f^{N}$ configuration for $n$ even) and the case of parity-forbidden $n$ photon transitions (as e.g. inter-configurational transitions from Stark levels of the $4 f^{N}$ configuration to Stark levels of the $4 f^{N-1} 5 d$ configuration for $n$ even).

\section{Introduction}

One- and two-photon spectroscopy [1-22] is a useful experimental tool for investigating the electronic properties of rare earth ions in crystals. The basic corresponding theoretical models have been developed by Judd [1] and Ofelt [2] for intra-configurational onephoton transitions (see [20] for a recent review) and by Axe [3] for intra-configurational two-photon transitions. The case of two-photon transitions has been the object of further works and extensions : (i) introduction of higher-order mechanisms for intraconfigurational transitions $[6,7,9,11]$, (ii) development of models for inter-configurational transitions $[10,12,15,21]$, and (iii) introduction of symmetry considerations from both a qualitative $[4,5]$ and a quantitative $[13,14,18,19,21,22]$ viewpoint.

In this paper, we present a theoretical model for $n$-photon dipolar transitions which includes, in a symmetry adapted form, the special cases $n=1[1,2]$ and $n=2[3]$. The model is developed for an arbitrary configuration of $N$ equivalent electrons in any symmetry $G$. Extensive use is made of symmetry adaptation techniques for the chain 
$O(3)^{*} \supset G^{*}$ (see [23]), where $G^{*}$ is the double group of the site symmetry group $G$.

The starting point of this work relies on the transition moment for an $n$-photon absorption between an initial state $i$ and a final state $f$. In the framework of the electric dipole approximation, it is given by the well-known formula

$$
M_{i \rightarrow f}=\frac{1}{\hbar^{n-1}} \sum_{\left\{v_{j}\right\}} \frac{\left(f\left|\left(\mathcal{E}_{1} \cdot D\right)\right| v_{1}\right)\left(v_{1}\left|\left(\mathcal{E}_{2} . D\right)\right| v_{2}\right) \cdots\left(v_{n-1}\left|\left(\mathcal{E}_{n} . D\right)\right| i\right)}{\left(\Omega_{i}-\Omega_{v_{1}}\right)\left(\Omega_{i}-\Omega_{v_{2}}\right) \cdots\left(\Omega_{i}-\Omega_{v_{n-1}}\right)}+\text { permutations }
$$

where the sum has to be extended over the intermediate states $v_{j}(j=1,2, \cdots, n-1)$ and permutations indicate that other terms must be added in order to take into account the $n$ ! permutations on the $n$ photons when they are different. In equation (1), the operator $\left(\mathcal{E}_{k} . D\right)$ stands for the scalar product of the polarization vector $\mathcal{E}_{k}$ of the $k$-th photon $(k=1,2, \cdots, n)$ with the dipole moment operator $D$ for the $N$ electrons. Furthermore, the energy denominators have their usual meaning.

At this stage, we have to make a sharp distinction between parity-allowed and parityforbidden $n$-photon transitions. We shall examine in turn (in sections 2 and 3 ) these two types of transitions. In section 4, we shall report on intensity formulas for parity-allowed and parity-forbidden transitions.

\section{Parity-allowed $n$-photon transitions}

In this case, the parity is a good quantum number for the initial, final and intermediate states. In other words, the initial and final states have the same parity for $n$ even and opposite parities for $n$ odd. Among the parity-allowed $n$-photon transitions, we thus may have intra-configurational transitions for $n$ even and inter-configurational transitions for $n$ odd. Two particular cases are of special interest, viz., the intra-configurational twophoton transitions $(n=2)$ and the inter-configurational one-photon transitions $(n=1)$.

For $n \neq 1$, the sum over $\left\{v_{j}\right\}$ in (1) is very difficult to handle since the energy denominators depend on the internal structure of the intermediate states. This situation is analogous to that encountered in the study of one-photon (parity-forbidden) intraconfigurational transitions. Therefore, we shall use here a quasi-closure approximation of the type introduced by Judd [1] and Ofelt [2] for $4 f^{N} \rightarrow 4 f^{N}$ one-photon transi- 
tions. As a net result, by using recoupling techniques in conjunction with a quasi-closure approximation, the transition matrix element (1) is amenable to the form

$$
M_{i \rightarrow f}=\left(f \Gamma^{\prime} \gamma^{\prime}\left|H_{\mathrm{eff}}\right| i \Gamma \gamma\right)
$$

with

$$
H_{\mathrm{eff}}=\sum_{\lambda_{1}, \lambda_{2}, \cdots, \lambda_{n-1}} C\left[\lambda_{1}, \lambda_{2}, \cdots, \lambda_{n-1}\right]\left(\left\{\cdots\left\{\left\{\mathcal{E}_{1} \mathcal{E}_{2}\right\}^{\lambda_{1}} \mathcal{E}_{3}\right\}^{\lambda_{2}} \cdots \mathcal{E}_{n}\right\}^{\lambda_{n-1}} \cdot U^{\lambda_{n-1}}\right)
$$

where (.) indicates a scalar product. In equation (2), the labels $\Gamma$ and $\Gamma^{\prime}$ stand for two irreducible representations of the group $G^{*}$. Furthermore, $\gamma$ and $\gamma^{\prime}$ are multiplicity labels. The labels $\Gamma \gamma$ and $\Gamma^{\prime} \gamma^{\prime}$ are the only good quantum numbers for the states $i$ and $f$, respectively. In equation (3), the electronic part is contained in the Racah unit tensor $U^{\lambda_{n-1}}$ while the polarization dependence is contained in the tensor \{\}$^{\lambda_{n-1}}$ (which describes the coupling of the $n$ polarization vectors associated with the $n$ photons). It should be noted that $\lambda_{n-1}$ cannot be equal to 0 (except for Rayleigh scattering). The $C$ parameters depend on the initial, final and intermediate configurations as well as the energies of the $n$ photons.

In the limiting case $n=1$, equation (3) yields the operator

$$
H_{\text {eff }}=(\mathcal{E} . D)
$$

of relevance for inter-configurational one-photon transitions. In the special case $n=2$, equation (3) returns the effective operator

$$
H_{\mathrm{eff}}=\sum_{\lambda=1,2} C[\lambda]\left(\left\{\mathcal{E}_{1} \mathcal{E}_{2}\right\}^{\lambda} \cdot U^{\lambda}\right)
$$

that describes intra-configurational two-photon transitions in the Axe model [3].

\section{Parity-forbidden $n$-photon transitions}

The $n$-photon transitions are forbidden (i.e., $M_{i \rightarrow f}=0$ ) between states of the same parity for $n$ odd and between states of opposite parities for $n$ even. However, such transi-

tions are observed in some cases. As an example, we have the case, treated independently 
by Judd [1] and Ofelt [2], of $4 f^{N} \rightarrow 4 f^{N}$ (intra-configurational) one-photon $(n=1)$ transitions. Indeed, such transitions become weakly allowed owing to a violation mechanism induced by the (static or dynamic) crystal-field interaction.

Going back to the case when $n$ is arbitrary, two parity violation mechanisms are possible in order to pass from $M_{i \rightarrow f}=0$ to $M_{i \rightarrow f} \neq 0$ :

(i) To use first-order time-independent perturbation theory in order to replace the initial, final and intermediate state vectors in (1) by state vectors with a not well-defined parity, the parity mixing being due to the odd crystal-field interaction.

(ii) To start from the transition matrix element for a parity-allowed $(n+1)$-photon transition and to replace one of the electric dipole operators $\left(\mathcal{E}_{k} . D\right)$ by the odd crystalfield interaction with appropriate permutations.

We shall refer the mechanisms described by (i) and (ii) to as $M[n, 1]$ and $M[n+1,0]$, respectively. The latter notation is a reminder that $M[n, 1]$, respectively $M[n+1,0]$, is concerned with $n^{\text {th }}$-order, respectively $(n+1)^{\text {th }}$-order, time-dependent perturbation theory and $1^{\text {st }}$-order, respectively $0^{\text {th }}$-order, time-independent perturbation theory. It can be shown that, under some approximation, we have $M[n+1,0] \approx M[n, 1]$ (see [21] for a detailed proof in the case $n=2$ ).

By using either the $M[n, 1]$ or $M[n+1,0]$ mechanism together with closure approximations and recoupling techniques, we can prove that the effective operator to be placed between the initial and the final $\left(0^{\text {th }}\right.$-order $)$ state vectors in equation $(2)$ reads

$$
H_{\mathrm{eff}}=\sum_{\lambda_{1}, \cdots, \lambda_{n}} \sum_{k} C\left[\lambda_{1}, \cdots, \lambda_{n-1} ; k ; \lambda_{n}\right]\left(\left\{\left\{\cdots\left\{\left\{\mathcal{E}_{1} \mathcal{E}_{2}\right\}^{\lambda_{1}} \mathcal{E}_{3}\right\}^{\lambda_{2}} \cdots \mathcal{E}_{n}\right\}^{\lambda_{n-1}} O^{k}\right\}^{\lambda_{n}} \cdot U^{\lambda_{n}}\right)
$$

Equation (6) should be compared with (3). In equation (6), the electronic part is described by the Racah unit tensor $U^{\lambda_{n}}$. The polarization information is contained in the coupled tensor \{\}$^{\lambda_{n}}$ where $O^{k}$ is a tensor whose components are defined from the odd crystal-field parameters [14]. The index $\lambda_{n-1}$ can take here the value 0 , in contradistinction to parity-allowed $n$-photon transitions. The $C$ parameters here parallel the ones 
occurring in (3) but are given by a different formula.

In the particular case $n=1$, equation (6) reads

$$
H_{\text {eff }}=\sum_{k \text { odd }} \sum_{\lambda \text { even }} C[k ; \lambda]\left(\left\{\mathcal{E} O^{k}\right\}^{\lambda} \cdot U^{\lambda}\right)
$$

which describes intra-configurational one-photon transitions, cf. $[1,2,14]$. For $n=2$, equation (6) reduces to the effective operator

$$
H_{\mathrm{eff}}=\sum_{\lambda_{1}=0,1,2} \sum_{k \text { odd }} \sum_{\lambda_{2}} C\left[\lambda_{1} ; k ; \lambda_{2}\right]\left(\left\{\left\{\mathcal{E}_{1} \mathcal{E}_{2}\right\}^{\lambda_{1}} O^{k}\right\}^{\lambda_{2}} \cdot U^{\lambda_{2}}\right)
$$

describing inter-configurational two-photon transitions [10,12,15,21].

\section{Intensity formula}

We are now in a position to give a formula for the intensity

$$
S_{\Gamma \rightarrow \Gamma^{\prime}}=\sum_{\gamma \gamma^{\prime}}\left|M_{i(\Gamma \gamma) \rightarrow f\left(\Gamma^{\prime} \gamma^{\prime}\right)}\right|^{2}
$$

of a (parity-allowed or parity-forbiddden) $n$-photon transition between the Stark levels $i$ of symmetry $\Gamma$ and $f$ of symmetry $\Gamma^{\prime}$. In equation (9), the sums over $\gamma$ and $\gamma^{\prime}$ have to be extended over all the components of the initial and final states, respectively.

The calculation of $S_{\Gamma \rightarrow \Gamma^{\prime}}$ may be achieved, by making use of symmetry adaptation techniques [23], in the following way : (i) express the initial and final states as well as the scalar products occurring in (3) and (6) in a form adapted to the chain of groups $O(3)^{*} \supset G^{*}$; (ii) apply the Wigner-Eckart theorem for the chain $O(3)^{*} \supset G^{*}$; (iii) use the factorization lemma for the coupling coefficients of the group $O(3)^{*}$ in an $O(3)^{*} \supset$ $G^{*}$ basis; and, finally (iv) use the so-called orthogonality-completeness property for the Clebsch-Gordan coefficients of the group $G$. We thus obtain the intensity formula

$$
S_{\Gamma \rightarrow \Gamma^{\prime}}=\sum_{\left\{k_{i}\right\}} \sum_{\left\{\ell_{i}\right\}} \sum_{r} \sum_{s} \sum_{\Gamma^{\prime \prime}} I\left[\left\{k_{i}\right\}\left\{\ell_{i}\right\} r s \Gamma^{\prime \prime} ; \Gamma \Gamma^{\prime}\right] \sum_{\gamma^{\prime \prime}} P_{r \Gamma^{\prime \prime} \gamma^{\prime \prime}}^{k_{n}-1}\left(P_{s \Gamma^{\prime \prime} \gamma^{\prime \prime}}^{\ell_{n}-1}\right)^{*}
$$

(with $1 \leq i \leq n-1$ ), the form of which holds for both parity-allowed and parity-forbidden

$n$-photon transitions. For a derivation of (10), the reader may consult [18] in the case of 
intra-configurational parity-allowed two-photon transitions and [21] in the case of interconfigurational parity-forbidden two-photon transitions.

The polarization dependence is clearly exhibited in (10) by the factors of type

$$
P^{\lambda_{n-1}}=\left\{\cdots\left\{\left\{\mathcal{E}_{1} \mathcal{E}_{2}\right\}^{\lambda_{1}} \mathcal{E}_{3}\right\}^{\lambda_{2}} \cdots \mathcal{E}_{n}\right\}^{\lambda_{n-1}}
$$

which already occur in (3) and (6). Of course, the intensity parameters $I$ are given by an expression that is specific of the kind of transition (parity-allowed or parity-forbidden) under consideration. The $I$ parameters depend on the initial and final states. They also depend on various reduced matrix elements ( \|\| ) of spherical tensors and on several energy parameters (for the involved configurations and the $n$ photons). In the case of parity-forbidden transitions, they depend on the odd crystal-field parameters.

The $I$ parameters present two interesting properties : (i) an hermitian conjugation property and (ii) a factorization property when the symmetry group $G$ is multiplicity-free [22]. The number of $I$ parameters in the intensity formula (10) is partially controlled by property (i) and the following group-theoretical selection rules : (i) the irreducible representation $\Gamma^{\prime \prime}$ of $G$ should be contained in the direct product $\Gamma^{* *} \otimes \Gamma$ and (ii) the irreducible representations $\left(k_{n-1}\right)$ and $\left(\ell_{n-1}\right)$ of $O(3)$ should contain $\Gamma^{\prime \prime}$. Let us also emphasize that the number of $I$ parameters depends as well on the number of absorbed photons and that the higher the symmetry, the lower the number of $I$ parameters.

\section{Discussion}

In this paper, we have concentrated on $n$-photon absorption between Stark levels, with well-defined symmetry species, for a transition ion (as, e.g., a rare earth ion) in finite symmetry. We have obtained intensity formulas, for both parity-allowed and parity-forbidden $n$-photon transitions, which incorporate all the information arising from symmetry considerations. In the particular case $n=2$, our results constitute a quantitative counterpart to the qualitative treatment in $[4,5]$. (By quantitative we mean that the intensity parameters are given by expressions arising from well-defined mechanisms and are calculable from first principles.) The latter point shows that the tables of Bader 
and Gold [5] can be used for inter-configurational two-photon transitions as well as for intra-configurational two-photon transitions.

The parity violation mechanism used in the present paper for parity-forbidden $n$ photon transitions is the same as the one introduced in the theory of Judd [1] and Ofelt [2] for intra-configurational $\left(4 f^{N} \rightarrow 4 f^{N}\right)$ one-photon transitions. Our treatment of parity-forbidden $n$-photon transitions is an extension (to $n$ arbitrary), in a symmetry adapted form, of the one by Judd and Ofelt.

For parity-allowed $n$-photon transitions, we have obtained results which extend, in a symmetry adapted form, the standard model of Axe [3] for intra-configurational twophoton transitions.

We know that for intra-configurational two-photon transitions, some additional mechanisms (additional with respect to the second-order mechanisms arising from secondorder time-dependent perturbation theory) have been introduced by various authors $[6,7,9,11]$ in order to produce more efficient third- and fourth-order mechanisms. For instance, the third-order mechanisms introduced by Judd and Pooler [6] arise from secondorder time-dependent perturbation theory plus first-order time-independent perturbation theory. Some additional mechanisms (including ligand-polarization effects) also may be introduced in the general case of parity-allowed and parity-forbidden $n$-photon transitions. This may lead to the replacement in equations (3) and (6) of the Racah unit tensor $U$ by some more complicated tensor with, for example, a spin- and orbit-dependence. The resulting intensity formula then assumes the same form as (10) (see [22]).

The intensity parameters $I$ in (10) (involving possibly additional mechanisms) can be calculated in principle in an ab initio way. This yields, however, a very intricate quantum chemistry problem which requires the knowledge of precise wave-functions. Therefore, it is often interesting to consider them, or part of them, as phenomenological parameters, at least in a first approach. In this connection, it should be emphasized that a phenomenological approach may incoporate various mechanisms in a global way. This may be an advantage, as well as an inconvenience because it is not always feasible to 
distinguish the contributions arising from various mechanisms.

Up to now, most of the experimental results for $n$-photon absorption are concerned with $n=1$ and 2. The formalism used in the present paper has been applied for $n=2$ to various rare earth and transition-metal ions in crystals (see $[13,16,17,19])$. We hope

that experimental data for polarization dependence of $n$-photon transitions will become available in order to test the model developed in this paper.

\section{Acknowledgements}

The authors are grateful to Dr. G.W. Burdick for a critical reading of the manuscript.

\section{References}

[1] B.R. Judd, Phys. Rev., 127 (1962) 750.

[2] G.S. Ofelt, J. Chem. Phys., 37 (1962) 511.

[3] J.D. Axe, Jr., Phys. Rev., 136 (1964) A42.

[4] M. Inoue and Y. Toyozawa, J. Phys. Soc. Japan, 20 (1965) 363.

[5] T.R. Bader and A. Gold, Phys. Rev., 171 (1968) 997.

[6] B.R. Judd and D.R. Pooler, J. Phys. C, 15 (1982) 591.

[7] M.C. Downer and A. Bivas, Phys. Rev. B, 28 (1983) 3677.

[8] S.K. Gayen and D.S. Hamilton, Phys. Rev. B, 28 (1983) 3706.

[9] M.F. Reid and F.S. Richardson, Phys. Rev. B, 29 (1984) 2830.

[10] R.C. Leavitt, Phys. Rev. B, 35 (1987) 9271.

[11] L. Smentek-Mielczarek and B.A. Hess, Jr., Phys. Rev. B, 36 (1987) 1811.

[12] A.G. Makhanek, V.S. Korolkov and L.A. Yuguryan, Phys. Status Solidi (b), 149 (1988) 231.

[13] J.C. Gâcon, J.F. Marcerou, M. Bouazaoui, B. Jacquier and M. Kibler, Phys. Rev. B, 40 (1989) 2070.

[14] M. Kibler and J.C. Gâcon, Croat. Chem. Acta, 62 (1989) 783.

[15] J. Sztucki and W. Strȩk, Chem. Phys., 143 (1990) 347.

[16] J.C. Gâcon, B. Jacquier, J.F. Marcerou, M. Bouazaoui and M. Kibler, J. Lumin., 45 (1990) 162.

[17] J.C. Gâcon, M. Bouazaoui, B. Jacquier, M. Kibler, L.A. Boatner and M.M. Abraham, Eur. J. Solid State Inorg. Chem., 28 (1991) 113.

[18] M.R. Kibler, in W. Florek, T. Lulek and M. Mucha (eds.), Symmetry and Structural Properties of Condensed Matter, World Scientific, Singapore, 1991, p. 237.

[19] J. Sztucki, M. Daoud and M. Kibler, Phys. Rev. B, 45 (1992) 2023.

[20] G.W. Burdick and M.C. Downer, in A.H. Kitai (ed.), Visible Luminescence, Chapman and Hall, London, in the press.

[21] M. Daoud and M. Kibler, Proc. International Workshop on Laser Physics, Dubna, Russia, April 1992, submitted for publication.

[22] M. Daoud, Doctorate Thesis, in preparation.

[23] M.R. Kibler, in J.C. Donini (ed.), Recent Advances in Group Theory and Their Application to Spectroscopy, Plenum Press, New York, 1979, p.1. 